\title{
max \\ Hadamard-Type Inequalities for Generalized Integral Operators Containing Special Functions
}

\author{
Chahnyong Jung ${ }^{1,+}+\mathbb{D}$, Ghulam Farid ${ }^{2, \dagger}$, Muhammad Yussouf ${ }^{3,+}$ and Kamsing Nonlaopon ${ }^{4, *,+}$ \\ 1 Department of Business Administration, Gyeongsang National University, Jinju 52828, Korea; \\ bb5734@gnu.ac.kr \\ 2 Department of Mathematics, Attock Campus, COMSATS University Islamabad, Attock 43600, Pakistan; \\ faridphdsms@hotmail.com \\ 3 Department of Mathematics, Talim-ul-Islam College, Chenab Nagar 35460, Pakistan; \\ mohsin2013.uos@gmail.com \\ 4 Department of Mathematics, Faculty of Science, Khon Kaen University, Khon Kaen 40002, Thailand \\ * Correspondence: nkamsi@kku.ac.th \\ $\dagger$ These authors contributed equally to this work.
}

check for updates

Citation: Jung, C.; Farid, G.; Yussouf, M.; Nonlaopon, K. Hadamard-Type Inequalities for Generalized Integral Operators Containing Special

Functions. Symmetry 2022, 14, 492. https://doi.org/10.3390/ sym 14030492

Academic Editor: Hüseyin Budak

Received: 14 January 2022

Accepted: 23 February 2022

Published: 28 February 2022

Publisher's Note: MDPI stays neutral with regard to jurisdictional claims in published maps and institutional affiliations.

Copyright: (C) 2022 by the authors. Licensee MDPI, Basel, Switzerland. This article is an open access article distributed under the terms and conditions of the Creative Commons Attribution (CC BY) license (https:// creativecommons.org/licenses/by/ $4.0 /)$.

\begin{abstract}
Convex functions are studied very frequently by means of the Hadamard inequality. A symmetric function leads to the generalization of the Hadamard inequality; the Fejér-Hadamard inequality is one of the generalizations of the Hadamard inequality that holds for convex functions defined on a finite interval along with functions which have symmetry about the midpoint of that finite interval. Lately, integral inequalities for convex functions have been extensively generalized by fractional integral operators. In this paper, inequalities of Hadamard type are generalized by using exponentially $(\alpha, h-m)$ - $p$-convex functions and an operator containing an extended generalized Mittag-Leffler function. The obtained results are also connected with several well-known Hadamardtype inequalities.
\end{abstract}

Keywords: Hadamard inequality; exponentially $(\alpha, h-m)-p$-convex function; generalized fractional integral operators; Mittag-Leffler function

\section{Introduction}

A well-known Mittag-Leffler function introduced in [1] generates exponential, trigonometric and hyperbolic functions. The role of the Mittag-Leffler function in fractional differential equations is important similar to the role of the exponential function in the ordinary and partial differential equations. In recent years, it has frequently been utilized to give the solutions of real-world problems studied in fractional calculus. For a detailed study on the applications of this special function see [2-5].

Formulating the classical concepts of calculus in the framework of fractional calculus has become a fashionable study nowadays. A lot of integral inequalities have been studied for fractional integrals and as a result, very interesting generalizations of classical inequalities can be found [6-9]. The Mittag-Leffler function is also used to define new generalized fractional integrals. For a detailed study of integral operators involving the Mittag-Leffler function, we refer the readers to [10-12] and references cited therein. The aim of this paper is to present Hadamard-type inequalities for fractional integrals containing an extended Mittag-Leffler function defined in [13]. To achieve these inequalities, a generalized class of functions named exponentially $(\alpha, h-m)$ - $p$-convex functions is utilized. Many well-known results are obtained in particular cases, as consequences of the main inequalities of this paper.

In the following, we give some important definitions and results, which are useful for the rest of this paper. The following integral operators are utilized to present the Hadamard-type inequalities. 
Definition 1. [13] Let $\omega, \tau, \delta, \rho, c \in \mathbb{C}, \Re(\tau), \Re(\delta)>0, \Re(c)>\Re(\rho)>0$ with $q \geq 0, \sigma, r>0$ and $0<k \leq r+\sigma$. Furthermore, let $f, g:[a, b] \rightarrow \mathbb{R}, 0<a<b$ be the two functions such that $f$ is positive and integrable and $g$ is differentiable and strictly increasing. Then, for $x \in[a, b]$ the integral operators are defined by:

$$
\begin{aligned}
& \left(g Y_{\sigma, \tau, \delta, \omega, a^{+}}^{\rho, r, k, c} f\right)(x ; q)=\int_{a}^{x}(g(x)-g(t))^{\tau-1} E_{\sigma, \tau, \delta}^{\rho, r, k}\left(\omega(g(x)-g(t))^{\sigma} ; q\right) f(t) d(g(t)), \\
& \left({ }_{g} Y_{\sigma, \tau, \delta, \delta, \omega, b^{-}}^{\rho, r, k} f\right)(x ; q)=\int_{x}^{b}(g(t)-g(x))^{\tau-1} E_{\sigma, \tau, \delta}^{\rho, r, k, c}\left(\omega(g(t)-g(x))^{\sigma} ; q\right) f(t) d(g(t)),
\end{aligned}
$$

where

$$
E_{\sigma, \tau, \delta}^{\rho, r, k, c}(t ; q)=\sum_{n=0}^{\infty} \frac{\beta_{q}(\rho+n k, c-\rho)(c)_{n k} t^{n}}{\beta(\rho, c-\rho) \Gamma(\sigma n+\tau)(\delta)_{n r}},
$$

is the generalized Mittag-Leffler function and $\beta_{q}$ is the extension of the beta function defined as follows:

$$
\beta_{q}(x, y)=\int_{0}^{1} t^{x-1}(1-t)^{y-1} e^{-\frac{q}{t(1-t)}} d t .
$$

Definition 2. [14] A real-valued function $f:[a, b] \rightarrow \mathbb{R}$ is called convex, if the following inequality holds:

$$
f(t x+(1-t) y) \leq t f(x)+(1-t) f(y), \forall x, y \in[a, b], t \in[0,1] .
$$

A convex function is equivalently studied by the well-known Hadamard inequality. The Hadamard inequality for convex functions was first generalized by Fejér using a symmetric function. The aim of this paper is to provide the generalizations of the Hadamard inequality for convex functions by using a class of functions that contains convex and many other related functions.

Next, we give some definitions of functions related to convex function that are useful for this study.

Definition 3. [15] A positive real-valued function $f: J \subset \mathbb{R} \rightarrow \mathbb{R}_{+}$is called exponentially convex on $J$, if the following inequality holds:

$$
f(t x+(1-t) y) \leq t f(x) / e^{\eta x}+(1-t) f(y) / e^{\eta y}, t \in[0,1], \forall x, y \in J, \eta \in \mathbb{R} .
$$

Definition 4. [16] Let $I \subset(0, \infty)$ be a real interval and $p \in \mathbb{R} \backslash\{0\}$. Then, a function $f: I \rightarrow \mathbb{R}$ is called $p$-convex, if the following inequality holds:

$$
f\left(\left(t x^{p}+(1-t) y^{p}\right)^{\frac{1}{p}}\right) \leq t f(x)+(1-t) f(y), \forall x, y \in I, t \in[0,1] .
$$

Definition 5. [17] Let $J \subseteq \mathbb{R}$ be an interval containing $(0,1)$ and let $h: J \rightarrow \mathbb{R}$ be a non-negative function. A function $f: I \rightarrow \mathbb{R}$ is called h-convex if the following inequality holds:

$$
f(t x+(1-t) y) \leq h(t) f(x)+h(1-t) f(y), \forall x, y \in I, t \in[0,1] .
$$

Definition 6. [14] A function $f:[0, b] \rightarrow \mathbb{R}, b>0$ is called $m$-convex, if the following inequality holds:

$$
f(t x+m(1-t) y) \leq t f(x)+m(1-t) f(y), \forall x, y \in[0, b], m, t \in[0,1] .
$$

Further extended definitions such as $(h-m)$-convex, $(s, m)$-convex, $(\alpha, m)$-convex, $(p, h)$-convex, $(\alpha, h-m)$-convex functions can be seen in [18-20]. All of the above definitions 
(2-6) and those mentioned in the previous sentence along with their exponentially convex cases can be observed in the following definition.

Definition 7. [21] Let $J \subseteq \mathbb{R}$ be an interval containing $(0,1)$ and let $h: J \rightarrow \mathbb{R}$ be a non-negative function. Let $I \subset(0, \infty)$ be a real interval and $p \in \mathbb{R} \backslash\{0\}$. A function $f: I \rightarrow \mathbb{R}$ is called exponentially $(\alpha, h-m)$-p-convex, if the following inequality holds:

$$
f\left(\left(t x^{p}+m(1-t) y^{p}\right)^{\frac{1}{p}}\right) \leq h\left(t^{\alpha}\right) f(x) / e^{\eta a}+m h\left(1-t^{\alpha}\right) f(y) / e^{\eta b},
$$

$\forall x, y \in I, \eta \in \mathbb{R},(\alpha, m) \in[0,1]^{2}, t \in[0,1]$.

For specific values of parameters and functions involved in Definition 7, one can get all the aforementioned definitions and types of functions. Furthermore, one can obtain the harmonically $h$-convex function (Definition 2.10) [22] by fixing $p=-1, m=\alpha=1$ and $\eta=0$, the exponentially (h-m)-p-convex function (Definition 13) [21] by fixing $\alpha=1$, the exponentially $(\alpha, m)$ - $p$-convex function (Definition 14) [21] by fixing $h(t)=t$ and the exponentially $(\alpha, h)$ - $p$-convex function (Definition 15) [21] by fixing $m=1$ in Definition 7. For more related definitions and functions, we refer the readers to (Remark 2.1) [21].

In the upcoming section, we prove two versions of the Hadamard inequality. Furthermore, results for the exponentially $(h-m)$ - $p$-convex, exponentially $(\alpha, m)$ - $p$-convex, exponentially $(\alpha, h)$ - $p$-convex and exponentially $(s, m)$-p-convex functions are explicitly given.

In the whole paper, we use the following notations for integral operators (1) and (2) as follows:

$$
\left(\mathcal{F}_{b, \sigma, \tau}^{a^{+}}\right)(\omega, f)=\left({ }_{g} \mathrm{Y}_{\sigma, \tau, \delta, \omega, a^{+}}^{\rho, r, k, c} f\right)(b ; q),\left(\mathcal{F}_{a, \sigma, \tau}^{b^{-}}\right)(\omega, f)=\left({ }_{g} \mathrm{Y}_{\sigma, \tau, \delta, \omega, \omega, b^{-}}^{\rho, r, k, c} f\right)(a ; q) .
$$

In the following, we use the notation $E_{p}(\alpha, h-m)$ for the exponentially $(\alpha, h-m)-p$ convex function.

\section{Hadamard-Type Inequalities}

In this section, we give Hadamard-type inequalities for $E_{p}(\alpha, h-m)$ functions via fractional integral operators containing Mittag-Leffler function. Furthermore, we discuss their connections to well-known results.

Theorem 1. Let $f, g:[a, b] \rightarrow \mathbb{R}, 0<a<b$ and range $(g) \subset[a, b]$ be the functions such that $f$ is positive and $f \in L_{1}[a, b], g$ is differentiable and strictly increasing. If $f$ is $E_{p}(\alpha, h-m)$ on $[a, b]$, then for integral operators (1) and (2), the following inequality holds:

(i) For $p>0$, then,

$$
\begin{aligned}
& f\left(\left(\frac{g^{p}(a)+m g^{p}(b)}{2}\right)^{\frac{1}{p}}\right)\left(\begin{array}{l}
\mathcal{F}_{g^{-1}\left(m g^{p}(b)\right), \sigma, \tau} \\
g^{-1}\left(g^{p}(a)\right)^{+}
\end{array}\right)\left(\omega^{\prime}, 1\right) \leq h\left(\frac{1}{2^{\alpha}}\right) \mathfrak{D}_{1}(\eta)\left(\mathcal{F}_{g^{-1}\left(m g^{p}(b)\right), \sigma, \tau}^{\left.g^{-1}\left(g^{p}\right)\right)^{+}}\right) \\
& \times\left(\omega^{\prime}, f \circ \zeta\right)+m^{\tau+1} h\left(\frac{2^{\alpha}-1}{2^{\alpha}}\right) \mathfrak{D}_{2}(\eta)\left(\begin{array}{c}
\mathcal{F}^{g^{-1}\left(g^{p}(b)\right)^{-}} \\
g^{-1}\left(\frac{g^{p}(a)}{m}\right), \sigma, \tau
\end{array}\right)\left(m^{\sigma} \omega^{\prime}, f \circ \zeta\right) \\
& \leq\left(h\left(\frac{1}{2^{\alpha}}\right) \mathfrak{D}_{1}(\eta) f(g(a)) / e^{\eta g(a)}+m h\left(\frac{2^{\alpha}-1}{2^{\alpha}}\right) \mathfrak{D}_{2}(\eta) f(g(b)) / e^{\eta g(b)}\right) \\
& \times \int_{0}^{1} t^{\tau-1} E_{\sigma, \tau, \delta}^{\rho, r, k, c}\left(\omega t^{\sigma} ; q\right) h\left(t^{\alpha}\right) d t+m\left(h\left(\frac{1}{2^{\alpha}}\right) \mathfrak{D}_{1}(\eta) f(g(b)) / e^{\eta g(b)}\right. \\
& \left.+m h\left(\frac{2^{\alpha}-1}{2^{\alpha}}\right) \mathfrak{D}_{2}(\eta) f\left(\frac{g(a)}{m^{2}}\right) / e^{\eta\left(g(a) / m^{2}\right)}\right) \int_{0}^{1} t^{\tau-1} E_{\sigma, \tau, \delta}^{\rho, r, k, c}\left(\omega t^{\sigma} ; q\right) h\left(1-t^{\alpha}\right) d t
\end{aligned}
$$


where $\zeta(z)=g^{\frac{1}{p}}(z), z \in\left[a^{p}, m b^{p}\right], \omega^{\prime}=\frac{\omega}{\left(m g^{p}(b)-g^{p}(a)\right)^{\sigma}}, \mathfrak{D}_{1}(\eta)=e^{-\eta g(b) m^{\frac{1}{p}}}$ for $\eta<0$, $\mathfrak{D}_{1}(\eta)=e^{-\eta g(a)}$ for $\eta \geq 0, \mathfrak{D}_{2}(\eta)=e^{-\eta\left(\frac{g(a)}{m^{\frac{1}{p}}}\right)}$ for $\eta>0, \mathfrak{D}_{2}(\eta)=e^{-\eta g(b)}$ for $\eta \leq 0$.

(ii) For $p<0$, then

$$
\begin{aligned}
& f\left(\left(\frac{g^{p}(a)+m g^{p}(b)}{2}\right)^{\frac{1}{p}}\right)\left(\mathcal{F}_{g^{-1}\left(m g^{p}(b)\right), \sigma, \tau}^{g^{-1}\left(g^{p}(a)\right)^{-}}\right)\left(\omega^{\prime}, 1\right) \leq h\left(\frac{1}{2^{\alpha}}\right) \mathfrak{D}_{3}(\eta)\left(\begin{array}{c}
\mathcal{F}_{g^{-1}\left(m g^{p}(b)\right), \sigma, \tau}^{\left.g^{-1}(a)\right)^{-}} \\
g^{-}
\end{array}\right) \\
& \times\left(\omega^{\prime}, f \circ \zeta\right)+m^{\tau+1} h\left(\frac{2^{\alpha}-1}{2^{\alpha}}\right) \mathfrak{D}_{4}(\eta)\left(\begin{array}{c}
\mathcal{F}^{g^{-1}\left(g^{p}(b)\right)^{+}} \\
g^{-1}\left(\frac{g^{p}(a)}{m}\right), \sigma, \tau
\end{array}\right)\left(m^{\sigma} \omega^{\prime}, f \circ \zeta\right) \\
& \leq\left(h\left(\frac{1}{2^{\alpha}}\right) \mathfrak{D}_{3}(\eta) f(g(a)) / e^{\eta g(a)}+m h\left(\frac{2^{\alpha}-1}{2^{\alpha}}\right) \mathfrak{D}_{4}(\eta) f(g(b)) / e^{\eta g(b)}\right) \\
& \times \int_{0}^{1} t^{\tau-1} E_{\sigma, \tau, \delta}^{\rho, r, k, c}\left(\omega t^{\sigma} ; q\right) h\left(t^{\alpha}\right) d t+m\left(h\left(\frac{1}{2^{\alpha}}\right) \mathfrak{D}_{3}(\eta) f(g(b)) / e^{\eta g(b)}\right. \\
& \left.+m h\left(\frac{2^{\alpha}-1}{2^{\alpha}}\right) \mathfrak{D}_{4}(\eta) f\left(\frac{g(a)}{m^{2}}\right) / e^{\eta\left(g(a) / m^{2}\right)}\right) \int_{0}^{1} t^{\tau-1} E_{\sigma, \tau, \delta}^{\rho, r, k, c}\left(\omega t^{\sigma} ; q\right) h\left(1-t^{\alpha}\right) d t,
\end{aligned}
$$

where $\zeta$ and $\omega^{\prime}$ are the same as given in Theorem $1(i), \mathfrak{D}_{3}(\eta)=e^{-\eta g(b) m^{\frac{1}{p}}}$ for $\eta>0, \mathfrak{D}_{3}(\eta)=$ $e^{-\eta g(a)}$ for $\eta \geq 0, \mathfrak{D}_{4}(\eta)=e^{-\eta\left(\frac{g(a)}{m^{\frac{1}{p}}}\right)}$ for $\eta<0, \mathfrak{D}_{4}(\eta)=e^{-\eta g(b)}$ for $\eta \geq 0$.

Proof. (i) By $E_{p}(\alpha, h-m)$ of $f$, one can observe

$$
f\left(\left(\frac{g^{p}(x)+m g^{p}(y)}{2}\right)^{\frac{1}{p}}\right) \leq h\left(\frac{1}{2^{\alpha}}\right) f(g(x)) / e^{\eta g(x)}+m h\left(\frac{2^{\alpha}-1}{2^{\alpha}}\right) f(g(y)) / e^{\eta g(y)} .
$$

By setting $g(x)=\left(\operatorname{tg}^{p}(a)+m(1-t) g^{p}(b)\right)^{\frac{1}{p}}$ and $g(y)=\left(\operatorname{tg}^{p}(b)+(1-t) \frac{g^{p}(a)}{m}\right)^{\frac{1}{p}}$ in (12) we get

$$
\begin{aligned}
& f\left(\left(\frac{g^{p}(a)+m g^{p}(b)}{2}\right)^{\frac{1}{p}}\right) \\
& \leq h\left(\frac{1}{2^{\alpha}}\right) \frac{f\left(\left(t^{p}(a)+m(1-t) g^{p}(b)\right)^{\frac{1}{p}}\right)}{\left.e^{\eta\left(\left(t^{p}(a)+m(1-t) g^{p}(b)\right)^{\frac{1}{p}}\right.}\right)}+m h\left(\frac{2^{\alpha}-1}{2^{\alpha}}\right) \frac{f\left(\left(\operatorname{tg} p(b)+(1-t) \frac{g^{p}(a)}{m}\right)^{\frac{1}{p}}\right)}{e^{\eta\left(\left(\operatorname{tg}^{p}(b)+(1-t) \frac{g^{p}(a)}{m}\right)^{\frac{1}{p}}\right)} .}
\end{aligned}
$$

Multiplying both sides of (13) by $t^{\tau-1} E_{\sigma, \tau, \delta}^{\rho, r, k, c}\left(\omega t^{\sigma} ; q\right)$ and integrating over $[0,1]$, we have

$$
\begin{aligned}
& f\left(\left(\frac{g^{p}(a)+m g^{p}(b)}{2}\right)^{\frac{1}{p}}\right) \int_{0}^{1} t^{\tau-1} E_{\sigma, \tau, \delta}^{\rho, r, k, c}\left(\omega t^{\sigma} ; q\right) d t \\
& \leq h\left(\frac{1}{2^{\alpha}}\right) \int_{0}^{1} \frac{t^{\tau-1} E_{\sigma, \tau, \delta}^{\rho, r, k, c}\left(\omega t^{\sigma} ; q\right) f\left(\left(\operatorname{tg}^{p}(a)+m(1-t) g^{p}(b)\right)^{\frac{1}{p}}\right)}{e^{\eta\left(\left(\operatorname{tg}^{p}(a)+m(1-t) g^{p}(b)\right)^{\frac{1}{p}}\right)}} d t \\
& +m h\left(\frac{2^{\alpha}-1}{2^{\alpha}}\right) \int_{0}^{1} \frac{t^{\tau-1} E_{\sigma, \tau, \delta}^{\rho, r, k, c}\left(\omega t^{\sigma} ; q\right) f\left(\left(\operatorname{tg}^{p}(b)+(1-t) \frac{g^{p}(a)}{m}\right)^{\frac{1}{p}}\right)}{e^{\eta\left(\left(\operatorname{tg}^{p}(b)+(1-t) \frac{g^{p}(a)}{m}\right)^{\frac{1}{p}}\right)}} d t .
\end{aligned}
$$


Setting $\operatorname{tg}^{p}(a)+m(1-t) g^{p}(b)=g(x)$, that is $t=\frac{m g^{p}(b)-x}{m g^{p}(b)-g^{p}(a)}$ and $\operatorname{tg}^{p}(b)+$ $(1-t) \frac{g^{p}(a)}{m}=g(y)$, that is $t=\frac{y-\frac{g^{p}(a)}{m}}{g^{p}(b)-\frac{g^{p}(a)}{m}}$ in (14), then, by using integral operators (1) and (2), the first inequality of (10) is obtained.

For the proof of the second inequality of (10), again by using $E_{p}(\alpha, h-m)$ of $f$, the right hand side of (13) leads to:

$$
\begin{aligned}
& h\left(\frac{1}{2^{\alpha}}\right) \frac{f\left(\left(\operatorname{tg}^{p}(a)+m(1-t) g^{p}(b)\right)^{\frac{1}{p}}\right)}{e^{\eta\left(\left(\operatorname{tg}^{p}(a)+m(1-t) g^{p}(b)\right)^{\frac{1}{p}}\right)}+m h\left(\frac{2^{\alpha}-1}{2^{\alpha}}\right) \frac{f\left(\left(\operatorname{tg}^{p}(b)+(1-t) \frac{g^{p}(a)}{m}\right)^{\frac{1}{p}}\right)}{\eta\left(\left(\operatorname{tg}^{p}(b)+(1-t) \frac{g^{p}(a)}{m}\right)^{\frac{1}{p}}\right)}} \\
& \leq \frac{e^{h\left(\frac{1}{2^{\alpha}}\right)}}{e^{\eta\left(\left(\operatorname{tg}^{p}(a)+m(1-t) g^{p}(b)\right)^{\frac{1}{p}}\right)}\left(h\left(t^{\alpha}\right) f(g(a)) / e^{\eta g(a)}+m h\left(1-t^{\alpha}\right) f(g(b)) / e^{\eta g(b)}\right)} \\
& +\frac{m h\left(\frac{2^{\alpha}-1}{2^{\alpha}}\right)}{e^{\eta\left(\left(g^{p}(b)+(1-t) \frac{g^{p}(a)}{m}\right)^{\frac{1}{p}}\right)}\left(h\left(t^{\alpha}\right) f(g(b)) / e^{\eta g(b)}+m h\left(1-t^{\alpha}\right) f\left(\frac{g(a)}{m^{2}}\right) / e^{\eta\left(g(a) / m^{2}\right)}\right) .}
\end{aligned}
$$

Multiplying $t^{\tau-1} E_{\sigma, \tau, \delta}^{\rho, r, k, c}\left(\omega t^{\sigma} ; q\right)$ on both sides of (15) and integrating over $[0,1]$, we have

$$
\begin{aligned}
& h\left(\frac{1}{2^{\alpha}}\right) \int_{0}^{1} \frac{t^{\tau-1} E_{\sigma, \tau, \delta}^{\rho, r, k, c}\left(\omega t^{\sigma} ; q\right) f\left(\left(\operatorname{tg}^{p}(a)+m(1-t) g^{p}(b)\right)^{\frac{1}{p}}\right)}{\eta\left(\left(g^{p}(a)+m(1-t) g^{p}(b)\right)^{\frac{1}{p}}\right)} d t \\
& +m h\left(\frac{2^{\alpha}-1}{2^{\alpha}}\right) \int_{0}^{1} \frac{t^{\tau-1} E_{\sigma, \tau, \delta}^{\rho, r, k, c}\left(\omega t^{\sigma} ; q\right) f\left(\left(t g^{p}(b)+(1-t) \frac{g^{p}(a)}{m}\right)^{\frac{1}{p}}\right)}{\eta\left(\left(g^{p}(b)+(1-t) \frac{g^{p}(a)}{m}\right)^{\frac{1}{p}}\right)} d t \\
& \leq\left(h\left(\frac{1}{2^{\alpha}}\right) \mathfrak{D}_{1}(\eta) f(g(a)) / e^{\eta g(a)}+m h\left(\frac{2^{\alpha}-1}{2^{\alpha}}\right) \mathfrak{D}_{2}(\eta) f(g(b)) / e^{\eta g(b)}\right) \\
& \times \int_{0}^{1} t^{\tau-1} E_{\sigma, \tau, \delta}^{\rho, r, k, c}\left(\omega t^{\sigma} ; q\right) h\left(t^{\alpha}\right) d t+m\left(h\left(\frac{1}{2^{\alpha}}\right) \mathfrak{D}_{1}(\eta) f(g(b)) / e^{\eta g(b)}\right. \\
& \left.+m h\left(\frac{2^{\alpha}-1}{2^{\alpha}}\right) \mathfrak{D}_{2}(\eta) f\left(\frac{g(a)}{m^{2}}\right) / e^{\eta\left(\frac{g(a)}{m^{2}}\right)}\right) \int_{0}^{1} t^{\tau-1} E_{\sigma, \tau, \delta}^{\rho, r, k, c}\left(\omega t^{\sigma} ; q\right) h\left(1-t^{\alpha}\right) d t .
\end{aligned}
$$

Setting $\operatorname{tg}^{p}(a)+m(1-t) g^{p}(b)=g(x)$, that is $t=\frac{m g^{p}(b)-x}{m g^{p}(b)-g^{p}(a)}$ and $\operatorname{tg}^{p}(b)+$ $(1-t) \frac{g^{p}(a)}{m}=g(y)$, that is $t=\frac{y-\frac{g^{p}(a)}{m}}{g^{p}(b)-\frac{g^{p}(a)}{m}}$ in (16), then, by using integral operators (1) and (2), the second inequality of $(10)$ is obtained.

(ii) The proof is similar to (i).

For different choices of parameter one can find a lot of existing results in the literature:

For example, (Theorem 2.2) [23] is obtained for $g=I, \eta=0$ and $\omega=q=0$ in Theorem 1(i), (Theorem 6) [13] is obtained by fixing $g=I, \eta=0$ and $\omega=q=0$ in Theorem 1(i), (Theorem 4) [6] is obtained by fixing $\alpha=m=1, p=-1, g=I$, $\omega=q=0, \eta=0$ and $h(t)=t$ in Theorem 1(ii), (Corollary 2.3) [6] is obtained by fixing $\alpha=m=1, p=-1, \zeta(z)=x, \eta=0$ and $h(t)=t$ in Theorem 1(ii), the classical Hadamard inequality [24,25] is obtained by fixing $\alpha=m=1, p=1, h(t)=t, \omega=q=0, \eta=0$ and $\tau=1$ in Theorem 1(i) and (Theorem 2) [8] is obtained by fixing $\alpha=m=1, h(t)=t, \eta=0$ and $p=1$ in Theorem 1(i). 
In the second result, we give another version of the Hadamard inequality for $E_{p}(\alpha, h-m)$ functions via fractional integral operators containing a Mittag-Leffler function.

Theorem 2. Suppose that the assumptions of Theorem 1 are valid. Then, we have:

(i) For $p>0$, then

$$
\begin{aligned}
& \left.f\left(\left(\frac{g^{p}(a)+m g^{p}(b)}{2}\right)^{\frac{1}{p}}\right)\left(\mathcal{F}_{g^{-1}\left(m g^{p}(b)\right), \sigma, \tau}^{g^{-1}\left(\frac{g^{p}(a)+m g^{p}(b)}{2}\right)^{+}}\right)\left(2^{\sigma} \omega^{\prime}, 1\right) \leq h\left(\frac{1}{2^{\alpha}}\right) \mathcal{D}_{1}(\eta)\left(\mathcal{F}_{g^{-1}\left(m g^{p}(b)\right), \sigma, \tau}^{\frac{g^{-1}(a)+m g^{p}(b)}{2}}\right)^{+}\right) \\
& \times\left(2^{\sigma} \omega^{\prime}, f \circ \zeta\right)+m^{\tau+1} h\left(\frac{2^{\alpha}-1}{2^{\alpha}}\right) \mathfrak{D}_{2}(\eta)\left(\mathcal{F}^{g^{-1}\left(\frac{g^{p}(a)+m g^{p}(b)}{2 m}\right)^{-1}}\right)\left((2 m)^{\sigma} \omega^{\prime}, f \circ \zeta\right) \\
& \leq\left(h\left(\frac{1}{2^{\alpha}}\right) \mathfrak{D}_{1}(\eta) f(g(a))+m h\left(\frac{2^{\alpha}-1}{2^{\alpha}}\right) \mathfrak{D}_{2}(\eta) f(g(b))\right) \int_{0}^{1} t^{\tau-1} E_{\sigma, \tau, \delta}^{\rho, r, k, c}\left(\omega t^{\sigma} ; q\right) h\left(\left(\frac{t}{2}\right)^{\alpha}\right) d t \\
& +m\left(h\left(\frac{1}{2^{\alpha}}\right) \mathfrak{D}_{1}(\eta) f(g(b))+m h\left(\frac{2^{\alpha}-1}{2^{\alpha}}\right) \mathfrak{D}_{2}(\eta) f\left(\frac{g(a)}{m^{2}}\right)\right) \int_{0}^{1} t^{\tau-1} E_{\sigma, \tau, \delta}^{\rho, r, k, c}\left(\omega t^{\sigma} ; q\right) h\left(1-\left(\frac{t}{2}\right)^{q}\right) d t,
\end{aligned}
$$

where $\zeta, \omega^{\prime}, \mathfrak{D}_{1}(\eta)$ and $\mathfrak{D}_{2}(\eta)$ are the same as in Theorem $1(i)$.

(ii) For $p<0$, then

$$
\begin{aligned}
& f\left(\left(\frac{g^{p}(a)+m g^{p}(b)}{2}\right)^{\frac{1}{p}}\right)\left(\mathcal{F}_{g^{-1}\left(m g^{p}(b)\right), \sigma, \tau}^{g^{-1}\left(\frac{g^{p}(a)+m g^{p}(b)}{2}\right)}\right)\left(2^{\sigma} \omega^{\prime}, 1\right) \leq h\left(\frac{1}{2^{\alpha}}\right) \mathfrak{D}_{3}(\eta)\left(\mathcal{F}_{g^{-1}\left(m g^{p}(b)\right), \sigma, \tau}^{g^{-1}\left(\frac{g^{p}(a)+m g^{p}(b)}{2}\right)-}\right) \\
& \times\left(2^{\sigma} \omega^{\prime}, f \circ \zeta\right)+m^{\tau+1} h\left(\frac{2^{\alpha}-1}{2^{\alpha}}\right) \mathfrak{D}_{4}(\eta)\left(\mathcal{F}^{g^{-1}\left(\frac{g^{p}(a)+m g^{p}(b)}{2 m}\right)}\right)\left((2 m)^{\sigma} \omega^{\prime}, f \circ \zeta\right) \\
& \leq\left(h\left(\frac{1}{2^{\alpha}}\right) \mathfrak{D}_{3}(\eta) f(g(a))+m h\left(\frac{2^{\alpha}-1}{2^{\alpha}}\right) \mathfrak{D}_{4}(\eta) f(g(b))\right) \int_{0}^{1} t^{\tau-1} E_{\sigma, \tau, \delta}^{\rho, r, k, c}\left(\omega t^{\sigma} ; q\right) h\left(\left(\frac{t}{2}\right)^{\alpha}\right) d t \\
& +m\left(h\left(\frac{1}{2^{\alpha}}\right) \mathfrak{D}_{3}(\eta) f(g(b))+m h\left(\frac{2^{\alpha}-1}{2^{\alpha}}\right) \mathfrak{D}_{4}(\eta) f\left(\frac{g(a)}{m^{2}}\right)\right) \int_{0}^{1} t^{\tau-1} E_{\sigma, \tau, \delta}^{\rho, r, k, c}\left(\omega t^{\sigma} ; q\right) h\left(1-\left(\frac{t}{2}\right)^{q}\right) d t,
\end{aligned}
$$

where $\zeta$ and $\omega^{\prime}$ are the same as in Theorem $1(i)$ and $\mathfrak{D}_{3}(\eta)$ and $\mathfrak{D}_{4}(\eta)$ are the same as in Theorem 1(ii).

Proof. (i) By setting $g(x)=\left(\frac{t}{2} g^{p}(a)+m\left(1-\frac{t}{2}\right) g^{p}(b)\right)^{\frac{1}{p}}$ and $g(y)=\left(\frac{t}{2} g^{p}(b)+\left(1-\frac{t}{2}\right)^{\frac{g^{p}}{m}(a)}\right)^{\frac{1}{p}}$ in (12) we get

$$
\begin{aligned}
& f\left(\left(\frac{g^{p}(a)+m g^{p}(b)}{2}\right)^{\frac{1}{p}}\right) \\
& \leq h\left(\frac{1}{2^{\alpha}}\right) \frac{f\left(\left(\frac{t}{2} g^{p}(a)+m\left(1-\frac{t}{2}\right) g^{p}(b)\right)^{\frac{1}{p}}\right)}{e^{\eta\left(\left(\frac{t}{2} g^{p}(a)+m\left(1-\frac{t}{2}\right) g^{p}(b)\right)^{\frac{1}{p}}\right)}}+m h\left(\frac{2^{\alpha}-1}{2^{\alpha}}\right) \frac{f\left(\left(\frac{t}{2} g^{p}(b)+\left(1-\frac{t}{2}\right) \frac{g^{p}(a)}{m}\right)^{\frac{1}{p}}\right)}{e^{\eta\left(\left(\frac{t}{2} g^{p}(b)+\left(1-\frac{t}{2}\right) \frac{g^{p}(a)}{m}\right)^{\frac{1}{p}}\right)} .}
\end{aligned}
$$

Multiplying both sides of (19) by $t^{\tau-1} E_{\sigma, \tau, \delta}^{\rho, r, k, c}\left(\omega t^{\sigma} ; q\right)$ and integrating over $[0,1]$, we have 


$$
\begin{aligned}
& f\left(\left(\frac{g^{p}(a)+m g^{p}(b)}{2}\right)^{\frac{1}{p}}\right) \int_{0}^{1} t^{\tau-1} E_{\sigma, \tau, \delta}^{\rho, r, k, c}\left(\omega t^{\sigma} ; q\right) d t \\
& \leq h\left(\frac{1}{2^{\alpha}}\right) \int_{0}^{1} \frac{t^{\tau-1} E_{\sigma, \tau, \delta}^{\rho, r, k, c}\left(\omega t^{\sigma} ; q\right) f\left(\left(\frac{t}{2} g^{p}(a)+m\left(1-\frac{t}{2}\right) g^{p}(b)\right)^{\frac{1}{p}}\right)}{\eta\left(\left(\frac{t}{2} g^{p}(a)+m\left(1-\frac{t}{2}\right) g^{p}(b)\right)^{\frac{1}{p}}\right)} d t \\
& +m h\left(\frac{2^{\alpha}-1}{2^{\alpha}}\right) \int_{0}^{1} \frac{t^{\tau-1} E_{\sigma, \tau, \delta}^{\rho, r, k, c}\left(\omega t^{\sigma} ; q\right) f\left(\left(\frac{t}{2} g^{p}(b)+\left(1-\frac{t}{2}\right) \frac{g^{p}(a)}{m}\right)^{\frac{1}{p}}\right)}{\eta\left(\left(\frac{t}{2} g^{p}(b)+\left(1-\frac{t}{2}\right) \frac{g^{p}(a)}{m}\right)^{\frac{1}{p}}\right)} d t . \\
& e^{\frac{1}{p}}
\end{aligned}
$$

Setting $\frac{t}{2} g^{p}(a)+m\left(1-\frac{t}{2}\right) g^{p}(b)=g(x)$, that is $\frac{t}{2}=\frac{m g^{p}(b)-x}{m g^{p}(b)-g^{p}(a)}$ and $\frac{t}{2} g^{p}(b)+$ $\left(1-\frac{t}{2}\right) \frac{g^{p}(a)}{m}=g(y)$, that is $\frac{t}{2}=\frac{y-\frac{g^{p}(a)}{m}}{g^{p}(b)-\frac{g^{p}(a)}{m}}$ in (20), then, by using integral operators (1) and (2), the first inequality of (17) is obtained.

For the proof of the second inequality of (17), again, by using $E_{p}(\alpha, h-m)$ of $f$, the right hand side of (19) leads to

$$
\begin{aligned}
& h\left(\frac{1}{2^{\alpha}}\right) \frac{f\left(\left(\frac{t}{2} g^{p}(a)+m\left(1-\frac{t}{2}\right) g^{p}(b)\right)^{\frac{1}{p}}\right)}{e^{\eta\left(\left(\frac{t}{2} g^{p}(a)+m\left(1-\frac{t}{2}\right) g^{p}(b)\right)^{\frac{1}{p}}\right)}}+m h\left(\frac{2^{\alpha}-1}{2^{\alpha}}\right) \frac{f\left(\left(\frac{t}{2} g^{p}(b)+\left(1-\frac{t}{2}\right) \frac{g^{p}(a)}{m}\right)^{\frac{1}{p}}\right)}{\eta\left(\left(\frac{t}{2} g^{p}(b)+\left(1-\frac{t}{2}\right) \frac{g^{p}(a)}{m}\right)^{\frac{1}{p}}\right)} \\
& \leq \frac{h\left(\frac{1}{2^{\alpha}}\right)}{e^{\eta\left(\left(\frac{t}{2} g^{p}(a)+m\left(1-\frac{t}{2}\right) g^{p}(b)\right)^{\frac{1}{p}}\right.}\left(h\left(\left(\frac{t}{2}\right)^{\alpha}\right) f(g(a)) / e^{\eta g(a)}+m h\left(1-\left(\frac{t}{2}\right)^{\alpha}\right) f(g(b)) / e^{\eta g(b)}\right)} \\
& +\frac{m h\left(\frac{2^{\alpha}-1}{2^{\alpha}}\right)}{\eta\left(\left(\frac{t}{2} g^{p}(b)+\left(1-\frac{t}{2}\right) \frac{g^{p}(a)}{m}\right)^{\frac{1}{p}}\right)}\left(h\left(\left(\frac{t}{2}\right)^{\alpha}\right) f(g(b)) / e^{\eta g(b)}+m h\left(1-\left(\frac{t}{2}\right)^{\alpha}\right) f\left(\frac{g(a)}{m^{2}}\right) / e^{\eta\left(g(a) / m^{2}\right)}\right) .
\end{aligned}
$$

Multiplying $t^{\tau-1} E_{\sigma, \tau, \delta}^{\rho, r, k, c}\left(\omega t^{\sigma} ; q\right)$ on both sides of (21) and integrating over $[0,1]$, we have

$$
\begin{aligned}
& h\left(\frac{1}{2^{\alpha}}\right) \int_{0}^{1} \frac{t^{\tau-1} E_{\sigma, \tau, \delta}^{\rho, r, k, c}\left(\omega t^{\sigma} ; q\right) f\left(\left(\frac{t}{2} g^{p}(a)+m\left(1-\frac{t}{2}\right) g^{p}(b)\right)^{\frac{1}{p}}\right)}{\left.e^{\eta\left(\left(\frac{t}{2} g^{p}(a)+m\left(1-\frac{t}{2}\right) g^{p}(b)\right)^{\frac{1}{p}}\right.}\right)} d t \\
& +m h\left(\frac{2^{\alpha}-1}{2^{\alpha}}\right) \int_{0}^{1} \frac{t^{\tau-1} E_{\sigma, \tau, \delta}^{\rho, r, k, c}\left(\omega t^{\sigma} ; q\right) f\left(\left(\frac{t}{2} g^{p}(b)+\left(1-\frac{t}{2}\right) \frac{g^{p}(a)}{m}\right)^{\frac{1}{p}}\right)}{\eta\left(\left(\frac{t}{2} g^{p}(b)+\left(1-\frac{t}{2}\right)_{\frac{g^{p}(a)}{m}}\right)^{\frac{1}{p}}\right)} d t \\
& \leq\left(h\left(\frac{1}{2^{\alpha}}\right) \mathfrak{D}_{1}(\eta) f(g(a)) / e^{\eta g(a)}+m h\left(\frac{2^{\alpha}-1}{2^{\alpha}}\right) \mathfrak{D}_{2}(\eta) f(g(b)) / e^{\eta g(b)}\right) \\
& \times \int_{0}^{1} t^{\tau-1} E_{\sigma, \tau, \delta}^{\rho, r, k, c}\left(\omega t^{\sigma} ; q\right) h\left(\left(\frac{t}{2}\right)^{\alpha}\right) d t+m\left(h\left(\frac{1}{2^{\alpha}}\right) \mathfrak{D}_{1}(\eta) f(g(b)) / e^{\eta g(b)}\right. \\
& \left.+m h\left(\frac{2^{\alpha}-1}{2^{\alpha}}\right) \mathfrak{D}_{2}(\eta) f\left(\frac{g(a)}{m^{2}}\right) / e^{\eta\left(\frac{g(a)}{m^{2}}\right)}\right) \int_{0}^{1} t^{\tau-1} E_{\sigma, \tau, \delta}^{\rho, r, k}\left(\omega t^{\sigma} ; q\right) h\left(1-\left(\frac{t}{2}\right)^{\alpha}\right) d t .
\end{aligned}
$$


Setting $\frac{t}{2} g^{p}(a)+m\left(1-\frac{t}{2}\right) g^{p}(b)=g(x)$, that is $\frac{t}{2}=\frac{m g^{p}(b)-x}{m g^{p}(b)-g^{p}(a)}$ and $\frac{t}{2} g^{p}(b)+$ $\left(1-\frac{t}{2}\right) \frac{g^{p}(a)}{m}=g(y)$, that is $\frac{t}{2}=\frac{y-\frac{g^{p}(a)}{m}}{g^{p}(b)-\frac{g^{p}(a)}{m}}$ in (22), then by using integral operators (1) and (2), the second inequality of (17) is obtained.

(ii) Proof is similar as (i).

For different choices of parameter one can observe a lot of results existing in the literature:

For example, (Theorem 2.4) [23] is obtained on fixing $g=I, \eta=0$ and $\omega=q=0$ in Theorem 2(i) and (Theorem 4) [7] is obtained on fixing $\alpha=1=m, p=-1, \omega=q=0$, $g=I, \eta=0$ and $h(t)=t$ in Theorem 2(ii).

Remark 1. From Theorems 1 and 2, one can deduce results for exponentially convex, exponentially $p$-convex, exponentially $m$-convex, exponentially $h$-convex, exponentially $(\alpha, m)$-convex, exponentially $(h-m)$-convex and exponentially $(p, h)$-convex functions, as well as all the results for operators given in Remark 1 [13].

\subsection{Results for Exponentially (h-m)-p-Convex Functions}

By setting $\alpha=1$ in Theorems 1 and 2, the results for exponentially (h-m)-p-convex functions $E_{p}(h-m)$ are obtained as follows:

Theorem 3. Suppose that the assumptions of Theorem 1 are valid. Then, for $E_{p}(h-m)$, we have:

(i) For $p>0$

$$
\begin{aligned}
& \frac{1}{h\left(\frac{1}{2}\right)} f\left(\left(\frac{g^{p}(a)+m g^{p}(b)}{2}\right)^{\frac{1}{p}}\right)\left(\mathcal{F}_{g^{-1}\left(m g^{p}(b)\right), \sigma, \tau}^{g^{-1}\left(g^{p}(a)\right)^{+}}\right)\left(\omega^{\prime}, 1\right) \\
& \leq \mathfrak{D}_{1}(\eta)\left(\mathcal{F}_{g^{-1}\left(m g^{p}(b)\right), \sigma, \tau}^{g^{-1}\left(g^{p}(a)\right)^{+}}\right)\left(\omega^{\prime}, f \circ \zeta\right)+m^{\tau+1} \mathfrak{D}_{2}(\eta)\left(\begin{array}{c}
\mathcal{F}^{g^{-1}\left(g^{p}(b)\right)^{-}} \\
g^{-1}\left(\frac{g^{p}(a)}{m}\right), \sigma, \tau
\end{array}\right)\left(m^{\sigma} \omega^{\prime}, f \circ \zeta\right) \\
& \leq\left(\mathfrak{D}_{1}(\eta) f(g(a))+m \mathfrak{D}_{2}(\eta) f(g(b))\right) \int_{0}^{1} t^{\tau-1} E_{\sigma, \tau, \delta}^{\rho, r, k, c}\left(\omega t^{\sigma} ; q\right) h(t) d t \\
& +m\left(\mathfrak{D}_{1}(\eta) f(g(b))+m \mathfrak{D}_{2}(\eta) f\left(\frac{g(a)}{m^{2}}\right)\right) \int_{0}^{1} t^{\tau-1} E_{\sigma, \tau, \delta}^{\rho, r, k, c}\left(\omega t^{\sigma} ; q\right) h(1-t) d t
\end{aligned}
$$

where $\zeta, \omega^{\prime}, \mathfrak{D}_{1}(\eta)$ and $\mathfrak{D}_{2}(\eta)$ are the same as in Theorem $1(i)$.

(ii) For $p<0$

$$
\begin{aligned}
& \frac{1}{h\left(\frac{1}{2}\right)} f\left(\left(\frac{g^{p}(a)+m g^{p}(b)}{2}\right)^{\frac{1}{p}}\right)\left(\begin{array}{c}
\mathcal{F}_{g^{-1}\left(m g^{p}(b)\right), \sigma, \tau}^{g^{-1}\left(g^{p}(a)\right.} \\
g^{-}
\end{array}\right)\left(\omega^{\prime}, 1\right) \\
& \leq \mathfrak{D}_{3}(\eta)\left(\mathcal{F}_{g^{-1}\left(g^{p}(a)\right)^{-}}^{-(b)), \sigma, \tau}\right)\left(\omega^{\prime}, f \circ \zeta\right)+m^{\tau+1} \mathfrak{D}_{4}(\eta)\left(\begin{array}{c}
\mathcal{F}^{g^{-1}\left(g^{p}(b)\right)^{+}} \\
g^{-1}\left(\frac{g^{p}(a)}{m}\right), \sigma, \tau
\end{array}\right)\left(m^{\sigma} \omega^{\prime}, f \circ \zeta\right) \\
& \leq\left(\mathfrak{D}_{3}(\eta) f(g(a))+m \mathfrak{D}_{4}(\eta) f(g(b))\right) \int_{0}^{1} t^{\tau-1} E_{\sigma, \tau, \delta}^{\rho, r, k, c}\left(\omega t^{\sigma} ; q\right) h(t) d t \\
& +m\left(\mathfrak{D}_{3}(\eta) f(g(b))+m \mathfrak{D}_{4}(\eta) f\left(\frac{g(a)}{m^{2}}\right)\right) \int_{0}^{1} t^{\tau-1} E_{\sigma, \tau, \delta}^{\rho, r, k, c}\left(\omega t^{\sigma} ; q\right) h(1-t) d t,
\end{aligned}
$$

where $\zeta$ and $\omega^{\prime}$ are the same as in Theorem $1(i)$ and $\mathfrak{D}_{3}(\eta)$ and $\mathfrak{D}_{4}(\eta)$ are the same as in Theorem 1(ii). 
Theorem 4. Suppose that the assumptions of Theorem 2 are valid. Then, for $E_{p}(h-m)$, we have:

(i) For $p>0$

$$
\begin{aligned}
& \frac{1}{h\left(\frac{1}{2}\right)} f\left(\left(\frac{g^{p}(a)+m g^{p}(b)}{2}\right)^{\frac{1}{p}}\right)\left(\mathcal{F}_{g^{-1}\left(m g^{p}(b)\right), \sigma, \tau}^{g^{-1}\left(\frac{g^{p}(a)+m g^{p}(b)}{2}\right)}\right)\left(2^{\sigma} \omega^{\prime}, 1\right) \\
& \leq \mathfrak{D}_{1}(\eta)\left(\mathcal{F}_{g^{-1}\left(m g^{p}(b)\right), \sigma, \tau}^{g^{-1}\left(\frac{g^{p}(a)+m g^{p}(b)}{2}\right)^{+}}\right)\left(2^{\sigma} \omega^{\prime}, f \circ \zeta\right)+m^{\tau+1} \mathfrak{D}_{2}(\eta)\left(\mathcal{F}_{g^{-1}\left(\frac{g^{p}(a)}{m}\right), \sigma, \tau}^{g^{-1}\left(\frac{g^{p}(a)+m g^{p}(b)}{2}\right)}\right)\left((2 m)^{\sigma} \omega^{\prime}, f \circ \zeta\right) \\
& \leq\left(\mathfrak{D}_{1}(\eta) f(g(a))+m \mathfrak{D}_{2}(\eta) f(g(b))\right) \int_{0}^{1} t^{\tau-1} E_{\sigma, \tau, \delta}^{\rho, r, k, c}\left(\omega t^{\sigma} ; q\right) h\left(\frac{t}{2}\right) d t \\
& +m\left(\mathfrak{D}_{1}(\eta) f(g(b))+m \mathfrak{D}_{2}(\eta) f\left(\frac{g(a)}{m^{2}}\right)\right) \int_{0}^{1} t^{\tau-1} E_{\sigma, \tau, \delta}^{\rho, r, k, c}\left(\omega t^{\sigma} ; q\right) h\left(1-\frac{t}{2}\right) d t,
\end{aligned}
$$

where $\zeta, \omega^{\prime}, \mathfrak{D}_{1}(\eta)$ and $\mathfrak{D}_{2}(\eta)$ are the same as in Theorem $1(i)$.

(ii) For $p<0$

$$
\begin{aligned}
& \frac{1}{h\left(\frac{1}{2}\right)} f\left(\left(\frac{g^{p}(a)+m g^{p}(b)}{2}\right)^{\frac{1}{p}}\right)\left(\mathcal{F}_{g^{-1}\left(m g^{p}(b)\right), \sigma, \tau}^{g^{-1}\left(\frac{g^{p}(a)+m g^{p}(b)}{2}\right)}\right)\left(2^{\sigma} \omega^{\prime}, 1\right) \\
& \leq \mathfrak{D}_{3}(\eta)\left(\mathcal{F}_{g^{-1}\left(m g^{p}(b)\right), \sigma, \tau}^{g^{-1}\left(\frac{g^{p}(a)+m g^{p}(b)}{2}\right)}\right)\left(2^{\sigma} \omega^{\prime}, f \circ \zeta\right)+m^{\tau+1} \mathfrak{D}_{4}(\eta)\left(\mathcal{F}_{g^{-1}\left(\frac{g^{p}(a)}{m}\right), \sigma, \tau}^{g^{-1}\left(\frac{g^{p}(a)+m g^{p}(b)}{2}\right)^{+}}\right)\left((2 m)^{\sigma} \omega^{\prime}, f \circ \zeta\right) \\
& \leq\left(\mathfrak{D}_{3}(\eta) f(g(a))+m \mathfrak{D}_{4}(\eta) f(g(b))\right) \int_{0}^{1} t^{\tau-1} E_{\sigma, \tau, \delta}^{\rho, r, k, c}\left(\omega t^{\sigma} ; q\right) h\left(\frac{t}{2}\right) d t \\
& +m\left(\mathfrak{D}_{3}(\eta) f(g(b))+m \mathfrak{D}_{4}(\eta) f\left(\frac{g(a)}{m^{2}}\right)\right) \int_{0}^{1} t^{\tau-1} E_{\sigma, \tau, \delta}^{\rho, r, k, c}\left(\omega t^{\sigma} ; q\right) h\left(1-\frac{t}{2}\right) d t,
\end{aligned}
$$

where $\zeta$ and $\omega^{\prime}$ are the same as in Theorem $1(i)$ and $\mathfrak{D}_{3}(\eta)$ and $\mathfrak{D}_{4}(\eta)$ are the same as in Theorem 1(ii).

\subsection{Results for Exponentially $(\alpha, m)$-p-Convex Functions}

By setting $h(t)=t$ in Theorems 1 and 2 , the results for exponentially $(\alpha, m)$ - $p$-convex functions $E_{p}(\alpha-m)$ are obtained as follows:

Theorem 5. Suppose that the assumptions of Theorem 1 are valid. Then, for $E_{p}(\alpha-m)$, we have:

$$
\text { (i) For } p>0
$$

$$
\begin{aligned}
& 2^{\alpha} f\left(\left(\frac{g^{p}(a)+m g^{p}(b)}{2}\right)^{\frac{1}{p}}\right)\left(\begin{array}{c}
\mathcal{F}_{g^{-1}\left(g^{p}(a)\right)^{+}}^{-1}\left(m g^{p}(b)\right), \sigma, \tau
\end{array}\right)\left(\omega^{\prime}, 1\right) \\
& \leq \mathfrak{D}_{1}(\eta)\left(\begin{array}{c}
\mathcal{F}_{g^{-1}\left(g^{p}(a)\right)^{+}}^{\left.g^{-1}(b)\right), \sigma, \tau}
\end{array}\right)\left(\omega^{\prime}, f \circ \zeta\right)+m^{\tau+1}\left(2^{\alpha}-1\right) \mathfrak{D}_{2}(\eta)\left(\begin{array}{c}
\mathcal{F}^{g^{-1}\left(g^{p}(b)\right)^{-}} \\
g^{-1}\left(\frac{g^{p}(a)}{m}\right), \sigma, \tau
\end{array}\right)\left(m^{\sigma} \omega^{\prime}, f \circ \zeta\right) \\
& \leq\left(\mathfrak{D}_{1}(\eta) f(g(a))+m\left(2^{\alpha}-1\right) \mathfrak{D}_{2}(\eta) f(g(b))\right)\left(\begin{array}{c}
\mathcal{F}^{g^{-1}\left(g^{p}(a)\right)^{+}} \\
g^{-1}\left(m g^{p}(b)\right), \sigma, \tau+\alpha
\end{array}\right)\left(\omega^{\prime}, 1\right) \\
& +m\left(\mathfrak{D}_{1}(\eta) f(g(b))+m\left(2^{\alpha}-1\right) \mathfrak{D}_{2}(\eta) f\left(\frac{g(a)}{m^{2}}\right)\right)\left(\left(\mathcal{F}_{g^{-1}(m g}^{g^{-1}\left(g^{p}(a)\right)^{+}}\right)\left(\omega^{\prime}, 1\right)\right. \\
& \left.-\left(\begin{array}{l}
\left.\mathcal{F}_{g^{-1}\left(g^{p}(a)\right)^{+}}^{+}(b)\right), \sigma, \tau+\alpha
\end{array}\right)\left(\omega^{\prime}, 1\right)\right)
\end{aligned}
$$


where $\zeta, \omega^{\prime}, \mathfrak{D}_{1}(\eta)$ and $\mathfrak{D}_{2}(\eta)$ are the same as in Theorem $1(i)$.

(ii) For $p<0$

$$
\begin{aligned}
& 2^{\alpha} f\left(\left(\frac{g^{p}(a)+m g^{p}(b)}{2}\right)^{\frac{1}{p}}\right)\left(\begin{array}{c}
\mathcal{F}_{g^{-1}\left(g^{p}(a)\right)^{-}}^{-1}\left(m g^{p}(b)\right), \sigma, \tau
\end{array}\right)\left(\omega^{\prime}, 1\right) \\
& \leq \mathfrak{D}_{3}(\eta)\left(\begin{array}{c}
\mathcal{F}_{g^{-1}\left(g^{p}(a)\right)^{-}}^{-1}\left(m g^{p}(b)\right), \sigma, \tau
\end{array}\right)\left(\omega^{\prime}, f \circ \zeta\right)+m^{\tau+1}\left(2^{\alpha}-1\right) \mathfrak{D}_{4}(\eta)\left(\begin{array}{c}
\mathcal{F}^{g^{-1}\left(g^{p}(b)\right)^{+}} \\
g^{-1}\left(\frac{g^{p}(a)}{m}\right), \sigma, \tau
\end{array}\right)\left(m^{\sigma} \omega^{\prime}, f \circ \zeta\right) \\
& \leq\left(\mathfrak{D}_{3}(\eta) f(g(a))+m\left(2^{\alpha}-1\right) \mathfrak{D}_{4}(\eta) f(g(b))\right)\left(\begin{array}{c}
\mathcal{F}_{g^{-1}}^{g^{-1}\left(g^{p}(a)\right)^{-}} \\
g^{-1}\left(m g^{p}(b)\right), \sigma, \tau+\alpha
\end{array}\right)\left(\omega^{\prime}, 1\right) \\
& +m\left(\mathfrak{D}_{3}(\eta) f(g(b))+m\left(2^{\alpha}-1\right) \mathfrak{D}_{4}(\eta) f\left(\frac{g(a)}{m^{2}}\right)\right)\left(\left(\mathcal{F}_{g^{-1}\left(m g^{-1}\left(g^{p}(a)\right), \sigma, \tau\right.}^{-}\right)\left(\omega^{\prime}, 1\right)\right. \\
& \left.-\left(\begin{array}{l}
\mathcal{F}_{g^{-1}}^{g^{-1}\left(g^{p}(a)\right)^{-}} \\
\left.g^{p}(b)\right), \sigma, \tau+\alpha
\end{array}\right)\left(\omega^{\prime}, 1\right)\right)
\end{aligned}
$$

where $\zeta$ and $\omega^{\prime}$ are the same as in Theorem $1(i)$ and $\mathfrak{D}_{3}(\eta)$ and $\mathfrak{D}_{4}(\eta)$ are the same as in Theorem 1(ii).

Theorem 6. Suppose that the assumptions of Theorem 2 are valid. Then, for $E_{p}(\alpha-m)$, we have: (i) For $p>0$

$$
\begin{aligned}
& 2^{\alpha} f\left(\left(\frac{g^{p}(a)+m g^{p}(b)}{2}\right)^{\frac{1}{p}}\right)\left(\mathcal{F}_{g^{-1}\left(m g^{p}(b)\right), \sigma, \tau}\right)^{-1}\left(\frac{g^{p}(a)+m g^{p}(b)}{2}\right)\left(2^{\sigma} \omega^{\prime}, 1\right) \\
& \leq \mathfrak{D}_{1}(\eta)\left(\mathcal{F}_{g^{-1}\left(m g^{p}(b)\right), \sigma, \tau}^{g^{-1}\left(\frac{g^{p}(a)+m g^{p}(b)}{2}\right)^{+}}\right)\left(2^{\sigma} \omega^{\prime}, f \circ \zeta\right)+m^{\tau+1}\left(2^{\alpha}-1\right) \mathfrak{D}_{2}(\eta)\left(\begin{array}{c}
\left.\mathcal{F}^{g^{-1}\left(\frac{g^{p}(a)+m g}{2 m}(b)\right.}\right)^{-} \\
g^{-1}\left(\frac{g^{p}(a)}{m}\right), \sigma, \tau
\end{array}\right)\left((2 m)^{\sigma} \omega^{\prime}, f \circ \zeta\right) \\
& \leq \frac{1}{2^{\alpha}}\left(\mathfrak{D}_{\mathbf{1}}(\eta) f(g(a))+m\left(2^{\alpha}-1\right) \mathfrak{D}_{2}(\eta) f(g(b))\right)\left(\begin{array}{c}
\mathcal{F}^{-1}\left(\frac{g^{p}(a)+m g^{p}(b)}{2}\right)^{+} \\
g^{-1}\left(m g^{p}(b)\right), \sigma, \tau+\alpha
\end{array}\right)\left(2^{\sigma} \omega^{\prime}, 1\right) \\
& +m\left(\mathfrak{D}_{1}(\eta) f(g(b))+m\left(2^{\alpha}-1\right) \mathfrak{D}_{2}(\eta) f\left(\frac{g(a)}{m^{2}}\right)\right)\left(\left(\mathcal{F}_{g^{-1}\left(m g^{p}(b)\right), \sigma, \tau}^{g^{-1}\left(\frac{g^{p}(a)+m g^{p}(b)}{2}\right)}\right)\left(2^{\sigma} \omega^{\prime}, 1\right)\right. \\
& \left.-\frac{1}{2^{\alpha}}\left(\mathcal{F}_{g^{-1}\left(m g^{p}(b)\right), \sigma, \tau+\alpha}^{g^{-1}\left(\frac{g^{p}(a)+m g^{p}(b)}{2}\right)^{+}}\right)\left(2^{\sigma} \omega^{\prime}, 1\right)\right)
\end{aligned}
$$

where $\zeta, \omega^{\prime}, \mathfrak{D}_{1}(\eta)$ and $\mathfrak{D}_{2}(\eta)$ are the same as in Theorem 1(i).

(ii) For $p<0$ 


$$
\begin{aligned}
& 2^{\alpha} f\left(\left(\frac{g^{p}(a)+m g^{p}(b)}{2}\right)^{\frac{1}{p}}\right)\left(\mathcal{F}_{g^{-1}\left(m g^{p}(b)\right), \sigma, \tau}^{g^{-1}\left(\frac{g^{p}(a)+m g^{p}(b)}{2}\right)^{-}}\right)\left(2^{\sigma} \omega^{\prime}, 1\right) \\
& \leq \mathfrak{D}_{3}(\eta)\left(\begin{array}{c}
\mathcal{F}_{g^{-1}\left(m g^{p}(b)\right), \sigma, \tau}^{g^{-1}\left(\frac{g^{p}(a)+m g^{p}(b)}{2}\right)} \\
g^{-}
\end{array}\right)\left(2^{\sigma} \omega^{\prime}, f \circ \zeta\right)+m^{\tau+1}\left(2^{\alpha}-1\right) \mathfrak{D}_{4}(\eta)\left(\begin{array}{c}
\mathcal{F}_{g^{-1}\left(\frac{g^{p}(a)}{m}\right), \sigma, \tau} g^{-1}\left(\frac{g^{p}(a)+m g^{p}(b)}{2 m}\right)^{+} \\
g^{-1}
\end{array}\right)\left((2 m)^{\sigma} \omega^{\prime}, f \circ \zeta\right) \\
& \leq \frac{1}{2^{\alpha}}\left(\mathfrak{D}_{3}(\eta) f(g(a))+m\left(2^{\alpha}-1\right) \mathfrak{D}_{4}(\eta) f(g(b))\right)\left(\mathcal{F}_{g^{-1}\left(m g^{p}(b)\right), \sigma, \tau+\alpha}^{g^{-1}\left(\frac{g^{p}(a)+m g^{p}(b)}{2}\right)^{-}}\right)\left(2^{\sigma} \omega^{\prime}, 1\right) \\
& +m\left(\mathfrak{D}_{3}(\eta) f(g(b))+m\left(2^{\alpha}-1\right) \mathfrak{D}_{4}(\eta) f\left(\frac{g(a)}{m^{2}}\right)\right)\left(\left(\begin{array}{c}
\mathcal{F}^{-1}\left(\frac{g^{p}(a)+m g^{p}(b)}{2}\right)^{-} \\
g^{-1}\left(m g^{p}(b)\right), \sigma, \tau
\end{array}\right)\left(2^{\sigma} \omega^{\prime}, 1\right)\right. \\
& \left.-\frac{1}{2^{\alpha}}\left(\mathcal{F}_{g^{-1}\left(m g^{p}(b)\right), \sigma, \tau+\alpha}^{g^{-1}\left(\frac{g^{p}(a)+m g^{p}(b)}{2}\right)^{-}}\right)\left(2^{\sigma} \omega^{\prime}, 1\right)\right)
\end{aligned}
$$

where $\zeta$ and $\omega^{\prime}$ are the same as in Theorem $1(i)$ and $\mathfrak{D}_{3}(\eta)$ and $\mathfrak{D}_{4}(\eta)$ are the same as in Theorem 1(ii).

\subsection{Results for Exponentially $(\alpha, h)$-p-Convex Functions}

By setting $m=1$ in Theorems 1 and 2, the results for exponentially $(\alpha, h)$ - $p$-convex functions $E_{p}(\alpha-h)$ are obtained as follows:

Theorem 7. Suppose that the assumptions of Theorem 1 are valid. Then, for $E_{p}(\alpha-h)$, we have:

$$
\text { (i) For } p>0
$$

$$
\begin{aligned}
& f\left(\left(\frac{g^{p}(a)+g^{p}(b)}{2}\right)^{\frac{1}{p}}\right)\left(\begin{array}{c}
\mathcal{F}^{g^{-1}\left(g^{p}(a)\right)^{+}} \\
g^{-1}\left(g^{p}(b)\right), \sigma, \tau
\end{array}\right)\left(\omega^{\prime}, 1\right) \\
& \leq h\left(\frac{1}{2^{\alpha}}\right) \mathfrak{D}_{1}(\eta)\left(\mathcal{F}_{g^{-1}\left(g^{-1}(b)\right), \sigma, \tau}^{\left.g^{p}(a)\right)^{+}}\right)\left(\omega^{\prime}, f \circ \zeta\right)+h\left(\frac{2^{\alpha}-1}{2^{\alpha}}\right) \mathfrak{D}_{2}(\eta)\left(\mathcal{F}_{g^{-1}\left(g^{-1}\left(g^{p}(b)\right), \sigma, \tau\right.}^{-}\right)\left(\omega^{\prime}, f \circ \zeta\right) \\
& \leq\left(h\left(\frac{1}{2^{\alpha}}\right) \mathfrak{D}_{1}(\eta) f(g(a))+h\left(\frac{2^{\alpha}-1}{2^{\alpha}}\right) \mathfrak{D}_{2}(\eta) f(g(b))\right) \int_{0}^{1} t^{\tau-1} E_{\sigma, \tau, \delta}^{\rho, r, k, c}\left(\omega t^{\sigma} ; q\right) h\left(t^{\alpha}\right) d t \\
& +\left(h\left(\frac{1}{2^{\alpha}}\right) \mathfrak{D}_{1}(\eta) f(g(b))+h\left(\frac{2^{\alpha}-1}{2^{\alpha}}\right) \mathfrak{D}_{2}(\eta) f(g(a))\right) \int_{0}^{1} t^{\tau-1} E_{\sigma, \tau, \delta}^{\rho, r, k, c}\left(\omega t^{\sigma} ; q\right) h\left(1-t^{\alpha}\right) d t
\end{aligned}
$$

where $\zeta, \omega^{\prime}, \mathfrak{D}_{1}(\eta)$ and $\mathfrak{D}_{2}(\eta)$ are the same as in Theorem 1(i).

(ii) For $p<0$

$$
\begin{aligned}
& f\left(\left(\frac{g^{p}(a)+g^{p}(b)}{2}\right)^{\frac{1}{p}}\right)\left(\begin{array}{c}
\mathcal{F}^{g^{-1}}\left(g^{p}(a)\right)^{-} \\
g^{-1}\left(g^{p}(b)\right), \sigma, \tau
\end{array}\right)\left(\omega^{\prime}, 1\right) \\
& \leq h\left(\frac{1}{2^{\alpha}}\right) \mathfrak{D}_{3}(\eta)\left(\mathcal{F}_{g^{-1}\left(g^{p}(b)\right), \sigma, \tau}^{g^{-1}\left(g^{p}(a)\right)^{-}}\right)\left(\omega^{\prime}, f \circ \zeta\right)+h\left(\frac{2^{\alpha}-1}{2^{\alpha}}\right) \mathfrak{D}_{4}(\eta)\left(\mathcal{F}_{g^{-1}\left(g^{p}(a)\right), \sigma, \tau}\right)\left(\omega^{\prime}, f \circ \zeta\right) \\
& \leq\left(h\left(\frac{1}{2^{\alpha}}\right) \mathfrak{D}_{3}(\eta) f(g(a))+h\left(\frac{2^{\alpha}-1}{2^{\alpha}}\right) \mathfrak{D}_{4}(\eta) f(g(b))\right) \int_{0}^{1} t^{\tau-1} E_{\sigma, \tau, \delta}^{\rho, r, k, c}\left(\omega t^{\sigma} ; q\right) h\left(t^{\alpha}\right) d t \\
& +\left(h\left(\frac{1}{2^{\alpha}}\right) \mathfrak{D}_{3}(\eta) f(g(b))+h\left(\frac{2^{\alpha}-1}{2^{\alpha}}\right) \mathfrak{D}_{4}(\eta) f(g(a))\right) \int_{0}^{1} t^{\tau-1} E_{\sigma, \tau, \delta}^{\rho, r, k, c}\left(\omega t^{\sigma} ; q\right) h\left(1-t^{\alpha}\right) d t
\end{aligned}
$$

where $\zeta$ and $\omega^{\prime}$ are the same as in Theorem $1(i)$ and $\mathfrak{D}_{3}(\eta)$ and $\mathfrak{D}_{4}(\eta)$ are the same as in Theorem 1(ii). 
Theorem 8. Suppose that the assumptions of Theorem 2 are valid. Then, for $E_{p}(\alpha-h)$, we have:

(i) For $p>0$

$$
\begin{aligned}
& \left.\left.f\left(\left(\frac{g^{p}(a)+g^{p}(b)}{2}\right)^{\frac{1}{p}}\right)\left(\mathcal{F}_{g^{-1}\left(g^{p}(b)\right), \sigma, \tau}^{-1}\right)^{g^{p}(a)+g^{p}(b)}\right)^{+}\right)\left(2^{\sigma} \omega^{\prime}, 1\right) \\
& \leq h\left(\frac{1}{2^{\alpha}}\right) \mathfrak{D}_{1}(\eta)\left(\mathcal{F}_{g^{-1}\left(g^{\left.g^{-1}(b)\right), \sigma, \tau}\left(\frac{g^{p}(a)+g^{p}(b)}{2}\right)^{+}\right.}\right)\left(2^{\sigma} \omega^{\prime}, f \circ \zeta\right)+h\left(\frac{2^{\alpha}-1}{2^{\alpha}}\right) \mathfrak{D}_{2}(\eta)\left(\mathcal{F}_{g^{-1}\left(g^{p}(a)\right), \sigma, \tau}^{g^{-1}\left(\frac{g^{p}(a)+g^{p}(b)}{2}\right)^{-}}\right)\left(2^{\sigma} \omega^{\prime}, f \circ \zeta\right) \\
& \leq\left(h\left(\frac{1}{2^{\alpha}}\right) \mathfrak{D}_{1}(\eta) f(g(a))+h\left(\frac{2^{\alpha}-1}{2^{\alpha}}\right) \mathfrak{D}_{2}(\eta) f(g(b))\right) \int_{0}^{1} t^{\tau-1} E_{\sigma, \tau, \delta}^{\rho, r, k, c}\left(\omega t^{\sigma} ; q\right) h\left(\left(\frac{t}{2}\right)^{\alpha}\right) d t \\
& +\left(h\left(\frac{1}{2^{\alpha}}\right) \mathfrak{D}_{1}(\eta) f(g(b))+h\left(\frac{2^{\alpha}-1}{2^{\alpha}}\right) \mathfrak{D}_{2}(\eta) f(g(a))\right) \int_{0}^{1} t^{\tau-1} E_{\sigma, \tau, \delta}^{\rho, r, k, c}\left(\omega t^{\sigma} ; q\right) h\left(1-\left(\frac{t}{2}\right)^{\alpha}\right) d t,
\end{aligned}
$$

where $\zeta, \omega^{\prime}, \mathfrak{D}_{1}(\eta)$ and $\mathfrak{D}_{2}(\eta)$ are the same as in Theorem $1(i)$.

(ii) For $p<0$

$$
\begin{aligned}
& \left.\left.f\left(\left(\frac{g^{p}(a)+g^{p}(b)}{2}\right)^{\frac{1}{p}}\right)\left(\mathcal{F}_{g^{-1}\left(g^{p}(b)\right), \sigma, \tau}^{-1}\right)^{g^{p}(a)+g^{p}(b)}\right)^{-}\right)\left(2^{\sigma} \omega^{\prime}, 1\right) \\
& \left.\leq h\left(\frac{1}{2^{\alpha}}\right) \mathfrak{D}_{3}(\eta)\left(\mathcal{F}_{g^{-1}\left(g^{\left.g^{-1}(b)\right), \sigma, \tau}\right.}^{\frac{g^{p}(a)+g^{p}(b)}{2}}\right)^{-}\right)\left(2^{\sigma} \omega^{\prime}, f \circ \zeta\right)+h\left(\frac{2^{\alpha}-1}{2^{\alpha}}\right) \mathfrak{D}_{4}(\eta)\left(\mathcal{F}_{g^{-1}\left(g^{p}(a)\right), \sigma, \tau}^{g^{-1}\left(\frac{g^{p}(a)+g^{p}(b)}{2}\right)^{+}}\right)\left(2^{\sigma} \omega^{\prime}, f \circ \zeta\right) \\
& \leq\left(h\left(\frac{1}{2^{\alpha}}\right) \mathfrak{D}_{3}(\eta) f(g(a))+h\left(\frac{2^{\alpha}-1}{2^{\alpha}}\right) \mathfrak{D}_{4}(\eta) f(g(b))\right) \int_{0}^{1} t^{\tau-1} E_{\sigma, \tau, \delta}^{\rho, r, k, c}\left(\omega t^{\sigma} ; q\right) h\left(\left(\frac{t}{2}\right)^{\alpha}\right) d t \\
& +\left(h\left(\frac{1}{2^{\alpha}}\right) \mathfrak{D}_{3}(\eta) f(g(b))+h\left(\frac{2^{\alpha}-1}{2^{\alpha}}\right) \mathfrak{D}_{4}(\eta) f(g(a))\right) \int_{0}^{1} t^{\tau-1} E_{\sigma, \tau, \delta}^{\rho, r, k, c}\left(\omega t^{\sigma} ; q\right) h\left(1-\left(\frac{t}{2}\right)^{\alpha}\right) d t,
\end{aligned}
$$

where $\zeta$ and $\omega^{\prime}$ are the same as in Theorem $1(i)$ and $\mathfrak{D}_{3}(\eta)$ and $\mathfrak{D}_{4}(\eta)$ are the same as in Theorem 1(ii).

\subsection{Results for Exponentially (s-m)-p-Convex Functions}

By setting $\alpha=1$ and $h(t)=t^{s}$ in Theorems 1 and 2, the results for exponentially $(s, m)$ - $p$-convex functions $E_{p}(s-m)$ are obtained as follows:

Theorem 9. Suppose that the assumptions of Theorem 1 are valid. Then, for $E_{p}(s-m)$, we have:

(i) For $p>0$

$$
\begin{aligned}
& 2^{s} f\left(\left(\frac{g^{p}(a)+m g^{p}(b)}{2}\right)^{\frac{1}{p}}\right)\left(\begin{array}{c}
\left.\mathcal{F}_{g^{-1}\left(g^{p}(a)\right)^{+}}^{-1}(b)\right), \sigma, \tau \\
g^{\prime}
\end{array}\right)\left(\omega^{\prime}, 1\right) \\
& \leq \mathfrak{D}_{1}(\eta)\left(\begin{array}{c}
\mathcal{F}_{g^{-1}\left(g^{p}(a)\right)^{+}} \\
g^{-1}\left(m g^{p}(b)\right), \sigma, \tau
\end{array}\right)\left(\omega^{\prime}, f \circ \zeta\right)+m^{\tau+1} \mathfrak{D}_{2}(\eta)\left(\begin{array}{c}
\mathcal{F}^{g^{-1}\left(g^{p}(b)\right)^{-}} \\
g^{-1}\left(\frac{g^{p}(a)}{m}\right), \sigma, \tau
\end{array}\right)\left(m^{\sigma} \omega^{\prime}, f \circ \zeta\right) \\
& \leq\left(\mathfrak{D}_{1}(\eta) f(g(a))+m \mathfrak{D}_{2}(\eta) f(g(b))\right)\left(\mathcal{F}_{g^{-1}\left(m g^{p}(b)\right), \sigma, \tau+s}^{g^{-1}\left(g^{p}(a)\right)^{+}}\right)\left(\omega^{\prime}, 1\right) \\
& +m\left(\mathfrak{D}_{1}(\eta) f(g(b))+m \mathfrak{D}_{2}(\eta) f\left(\frac{g(a)}{m^{2}}\right)\right) \int_{0}^{1} t^{\tau-1}(1-t)^{s} E_{\sigma, \tau, \delta}^{\rho, r, k, c}\left(\omega t^{\sigma} ; q\right) d t,
\end{aligned}
$$

where $\zeta, \omega^{\prime}, \mathfrak{D}_{1}(\eta)$ and $\mathfrak{D}_{2}(\eta)$ are the same as in Theorem 1(i). 
(ii) For $p<0$

$$
\begin{aligned}
& 2^{s} f\left(\left(\frac{g^{p}(a)+m g^{p}(b)}{2}\right)^{\frac{1}{p}}\right)\left(\begin{array}{c}
\mathcal{F}_{g^{-1}\left(g^{p}(a)\right)^{-}}^{-1}\left(m g^{p}(b)\right), \sigma, \tau
\end{array}\right)\left(\omega^{\prime}, 1\right) \\
& \leq \mathfrak{D}_{3}(\eta)\left(\begin{array}{c}
\mathcal{F}_{g^{-1}\left(g^{p}(a)\right)^{-}}\left(m g^{p}(b)\right), \sigma, \tau
\end{array}\right)\left(\omega^{\prime}, f \circ \zeta\right)+m^{\tau+1} \mathfrak{D}_{4}(\eta)\left(\begin{array}{c}
\mathcal{F}^{g^{-1}\left(g^{p}(b)\right)^{+}} \\
g^{-1}\left(\frac{g^{p}(a)}{m}\right), \sigma, \tau
\end{array}\right)\left(m^{\sigma} \omega^{\prime}, f \circ \zeta\right) \\
& \leq\left(\mathfrak{D}_{3}(\eta) f(g(a))+m \mathfrak{D}_{4}(\eta) f(g(b))\right)\left(\begin{array}{c}
\mathcal{F}^{g^{-1}\left(g^{p}(a)\right)^{-}} \\
g^{-1}\left(m g^{p}(b)\right), \sigma, \tau+s
\end{array}\right)\left(\omega^{\prime}, 1\right) \\
& +m\left(\mathfrak{D}_{3}(\eta) f(g(b))+m \mathfrak{D}_{4}(\eta) f\left(\frac{g(a)}{m^{2}}\right)\right) \int_{0}^{1} t^{\tau-1}(1-t)^{s} E_{\sigma, \tau, \delta}^{\rho, r, k, c}\left(\omega t^{\sigma} ; q\right) d t,
\end{aligned}
$$

where $\zeta$ and $\omega^{\prime}$ are the same as in Theorem $1(i)$ and $\mathfrak{D}_{3}(\eta)$ and $\mathfrak{D}_{4}(\eta)$ are the same as in Theorem 1(ii).

Theorem 10. Suppose that the assumptions of Theorem 2 are valid. Then, for $E_{p}(s-m)$, we have:

$$
\text { (i) For } p>0
$$

$$
\begin{aligned}
& 2^{s} f\left(\left(\frac{g^{p}(a)+m g^{p}(b)}{2}\right)^{\frac{1}{p}}\right)\left(\mathcal{F}_{g^{-1}\left(m g^{p}(b)\right), \sigma, \tau}^{g^{-1}\left(\frac{g^{p}(a)+m g^{p}(b)}{2}\right)^{+}}\right)\left(2^{\sigma} \omega^{\prime}, 1\right) \\
& \leq \mathfrak{D}_{1}(\eta)\left(\begin{array}{c}
\mathcal{F}_{\left.g^{-1}\left(\frac{g^{p}(a)+m g^{p}(b)}{2}\right)^{+}(b)\right), \sigma, \tau}^{+}
\end{array}\right)\left(2^{\sigma} \omega^{\prime}, f \circ \zeta\right)+m^{\tau+1} \mathfrak{D}_{2}(\eta)\left(\begin{array}{c}
\mathcal{F}^{g^{-1}\left(\frac{g^{p}(a)+m g^{p}(b)}{2 m}\right)^{-}} \\
g^{-1}\left(\frac{g^{p}(a)}{m}\right), \sigma, \tau
\end{array}\right)\left((2 m)^{\sigma} \omega^{\prime}, f \circ \zeta\right) \\
& \leq \frac{1}{2^{s}}\left(\mathfrak{D}_{1}(\eta) f(g(a))+m \mathfrak{D}_{2}(\eta) f(g(b))\right)\left(\mathcal{F}_{g^{-1}\left(m g^{p}(b)\right), \sigma, \tau+s}^{g^{-1}\left(\frac{g^{p}(a)+m g^{p}(b)}{2}\right)^{+}}\right)\left(2^{\sigma} \omega^{\prime}, 1\right) \\
& +m\left(\mathfrak{D}_{1}(\eta) f(g(b))+m \mathfrak{D}_{2}(\eta) f\left(\frac{g(a)}{m^{2}}\right)\right) \int_{0}^{1} t^{\tau-1}\left(1-\frac{t}{2}\right)^{s} E_{\sigma, \tau, \delta}^{\rho, r, k, c}\left(\omega t^{\sigma} ; q\right) d t,
\end{aligned}
$$

where $\zeta, \omega^{\prime}, \mathfrak{D}_{1}(\eta)$ and $\mathfrak{D}_{2}(\eta)$ are the same as in Theorem $1(i)$.

(ii) For $p<0$

$$
\begin{aligned}
& 2^{s} f\left(\left(\frac{g^{p}(a)+m g^{p}(b)}{2}\right)^{\frac{1}{p}}\right)\left(\mathcal{F}_{g^{-1}\left(m g^{p}(b)\right), \sigma, \tau}^{g^{-1}\left(\frac{g^{p}(a)+m g^{p}(b)}{2}\right)^{-}}\right)\left(2^{\sigma} \omega^{\prime}, 1\right) \\
& \leq \mathfrak{D}_{3}(\eta)\left(\mathcal{F}_{g^{-1}\left(m g^{p}(b)\right), \sigma, \tau}^{g^{-1}\left(\frac{g^{p}(a)+m g^{p}(b)}{2}\right)}\right)\left(2^{\sigma} \omega^{\prime}, f \circ \zeta\right)+m^{\tau+1} \mathfrak{D}_{4}(\eta)\left(\mathcal{F}_{g^{-1}\left(\frac{g^{p}(a)}{m}\right), \sigma, \tau}^{g^{-1}\left(\frac{g^{p}(a)+m g^{p}(b)}{2 m}\right)^{+}}\right)\left((2 m)^{\sigma} \omega^{\prime}, f \circ \zeta\right) \\
& \leq \frac{1}{2^{s}}\left(\mathfrak{D}_{3}(\eta) f(g(a))+m \mathfrak{D}_{4}(\eta) f(g(b))\right)\left(\mathcal{F}_{g^{-1}\left(m g^{p}(b)\right), \sigma, \tau+s}^{g^{-1}\left(\frac{g^{p}(a)+m g^{p}(b)}{2}\right)^{-}}\right)\left(2^{\sigma} \omega^{\prime}, 1\right) \\
& +m\left(\mathfrak{D}_{3}(\eta) f(g(b))+m \mathfrak{D}_{4}(\eta) f\left(\frac{g(a)}{m^{2}}\right)\right) \int_{0}^{1} t^{\tau-1}\left(1-\frac{t}{2}\right)^{s} E_{\sigma, \tau, \delta}^{\rho, r, k, c}\left(\omega t^{\sigma} ; q\right) d t
\end{aligned}
$$

where $\zeta$ and $\omega^{\prime}$ are the same as in Theorem $1(i)$ and $\mathfrak{D}_{3}(\eta)$ and $\mathfrak{D}_{4}(\eta)$ are the same as in Theorem 1(ii).

Remark 2. From Theorems 1 and 2, one can deduce results for exponentially (s, $m)$-p-GodunovaLevin-convex function of the second kind, exponentially $(p, P)$-convex function, exponentially Godunova-Levin-type harmonic convex function, exponentially s-Godunova-Levin-type harmonic convex function, exponentially $(\alpha, h-m)-H A$-convex function, exponentially $(\alpha, h)-H A$-convex 
function, exponentially $H A$-convex function and exponentially $(\alpha, m)$-HA-convex function, as well as all the results for operators given in Remark 1 [13].

\section{Conclusions}

Generalized Hamamard-type fractional integral inequalities for exponentially $(\alpha, h, m)$ $p$-convex functions were presented. To achieve these results, we applied the generalized fractional integral operators containing an extended generalized Mittag-Leffler function. In particular cases, the Hadamard-type inequalities for exponentially $(h, m)$-p-convex functions, exponentially $(\alpha, m)$-p-convex functions, exponentially $(\alpha, h)$-p-convex functions and exponentially $(s, m)-p$-convex functions were deduced. Moreover, the connection with Hadamard-type inequalities for several types of convex functions was shown in Remark 2.

Author Contributions: Conceptualization, C.J., G.F., M.Y. and K.N.; Investigation, M.Y. and K.N.; Methodology, C.J.; Supervision, G.F.; Writing—original draft, M.Y.; Writing—review \& editing, C.J., G.F. and K.N. All authors have read and agreed to the published version of the manuscript.

Funding: This research received no external funding.

Institutional Review Board Statement: Not applicable.

Informed Consent Statement: Not applicable.

Data Availability Statement: Not applicable.

Acknowledgments: This work was supported by development fund foundation, Gyeongsang National University, 2021.

Conflicts of Interest: The authors declare no conflict of interest.

\section{References}

1. Mittag-Leffler, G.M. Sur la nouvelle fonction $E_{\alpha}(x)$. Comptes Rendus Acad. Sci. Paris 1903, 137, 554-558.

2. Giusti, A.; Colombaro, I. Prabhakar-like fractional viscoelasticity. Commun. Nonlinear Sci. Numer. Simul. 2018, 56, 138-143. [CrossRef]

3. Gorenflo, R.; Kilbas, A.A.; Mainardi, F.; Rogosin, S.V. Mittag-Leffler Functions, Related Topics and Applications; Springer: Berlin/Heidelberg, Germany, 2016.

4. Haubold, H.J.; Mathai, A.M.; Saxena, R.K. Mittag-Leffler functions and their applications. J. Appl. Math. 2011, 2011, 51 . [CrossRef]

5. Rogosin, S.V. The role of the Mittag-Leffler function in fractional modeling. J. Math. 2015, 3, 368-381. [CrossRef]

6. İscan, İ; Wu, S. Hermite-Hadamard type inequalities for harmonically convex functions via fractional integrals. Appl. Math. Comput. 2014, 238, 237-244.

7. Kunt, M.; İscan, İ.; Yazi, N.; Gozutok, U. On new inequalities of Hermite-Hadamard-Fejér type inequalities for harmonically convex functions via fractional integrals. Springer Plus 2016, 5, 1-19. [CrossRef] [PubMed]

8. Sarikaya, M.Z.; Set, E.; Yaldiz, H.; Basak, N. Hermite-Hadamard inequalities for fractional integrals and related fractional inequalities. J. Math. Comput. Model. 2013, 57, 2403-2407. [CrossRef]

9. Ahmad, B.; Alsaedi, A.; Kirane, M.; Torebek, B.T. Hermite-Hadamard, Hermite-Hadamard-Fejér, Dragomir-Agarwal and Pachpatte type inequalities for convex functions via new fractional integrals. J. Comp. Appl. Math. 2019, 353, 120-129. [CrossRef]

10. Prabhakar, T.R. A singular integral equation with a generalized Mittag-Leffler function in the kernel. Yokohama Math. J. 1971, 19, 7-15.

11. Salim, T.O.; Faraj, A.W. A generalization of Mittag-Leffler function and integral operator associated with integral calculus. J. Frac. Calc. Appl. 2012, 3, 1-13.

12. Srivastava, H.M. Some families of Mittag-Leffler type functions and associated operators of fractional calculus. TWMS J. Pure Appl. Math. 2016, 7, 123-145.

13. Rao, Y.; Yussouf, M.; Farid, G.; Pečarić, J.; Tlili, I. Further generalizations of Hadamard and Fejér-Hadamard inequalities and error estimations. Adv. Diff. Equ. 2020, 2020, 421. [CrossRef]

14. Toader, G.H. Some Generalization of Convexity. In Proceedings of the Colloquium on Approximation and Optimization, Cluj-Napoca, Romania, 25-27 October 1984; pp. 329-338.

15. Awan, M.U.; Noor, M.A.; Noor, K.I. Hermite-Hadamard inequalities for exponentially convex functions. Appl. Math. Inform. Sci. 2018, 12, 404-409. [CrossRef]

16. Zhang, K.S.; Wan, J.P. p-convex functions and their properties. Pure Appl. Math. 2007, 23, 130-133.

17. Varošanec, S. On h-convexity, J. Math. Anal. Appl. 2007, 326, 303-311. [CrossRef]

18. Fang, Z.B.; Shi, R. On the $(p, h)$-convex function and some integral inequalities. J. Inequal. Appl. 2014, 2014, 16 . [CrossRef]

19. Park, J. New Ostrowski-Like type inequalities for differentiable (s,m)-convex mappings. Int. J. Pure App. Math. 2012, 78, 1077-1089. 
20. Rehman, A.U.; Farid, G.; Ain, Q.U. Hermite-Hadamard type inequalities for (h-m)-convexity. Electron. J. Math. Anal. Appl. 2018, 6, 317-329.

21. Zhang, X.; Farid, G.; Yussouf, M.; Nonloapon, K. New generalized versions of Hadamard and Fejér-Hadamard inequalities for exponentially $(\alpha, h-m)$-p-Convex functions via Riemann-Liouville fractional integrals. submitted.

22. Noor, M.A.; Noor, K.I.; Awan, M.U.; Costaache, S. Some integral inequalities for harmonically $h$-convex functions. UPB Sci. Bull. Ser. A 2015, 77, 5-16.

23. Jia, W.; Yussouf, M.; Farid, G.; Khan, K.A. Hadamard and Fejér-Hadamard inequalities for $(\alpha, h-m)$ - $p$-Convex functions via Riemann-Liouville fractional integrals. Math. Probl. Eng. 2021, 2021, 12. [CrossRef]

24. Hermite, C. Sur deux limites d'une intgrale dfinie. Mathesis 1883, 3, 82.

25. Hadamard, J. Etude sur les proprietes des fonctions entieres e.t en particulier dune fonction consideree par Riemann. J. Math. Pure Appl. 1893, 58, 171-215. 